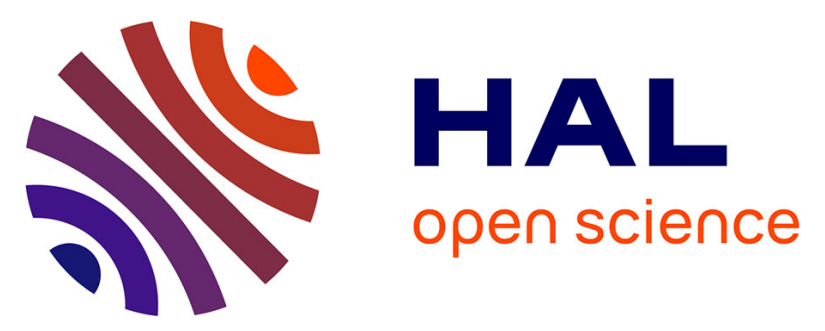

\title{
Lentiviral Expression of Rabies Virus Glycoprotein in the Rat Hippocampus Strengthens Synaptic Plasticity
}

Soheil Ghassemi, Tara Asgari, Hadi Mirzapour-Delavar, Shayan Aliakbari, Hamid Gholami Pourbadie, Christophe Prehaud, Monique Lafon, Alireza Gholami, Kayhan Azadmanesh, Nima Naderi, et al.

\section{To cite this version:}

Soheil Ghassemi, Tara Asgari, Hadi Mirzapour-Delavar, Shayan Aliakbari, Hamid Gholami Pourbadie, et al.. Lentiviral Expression of Rabies Virus Glycoprotein in the Rat Hippocampus Strengthens Synaptic Plasticity. 2021. pasteur-03190429

\section{HAL Id: pasteur-03190429}

\section{https://hal-pasteur.archives-ouvertes.fr/pasteur-03190429}

Preprint submitted on 6 Apr 2021

HAL is a multi-disciplinary open access archive for the deposit and dissemination of scientific research documents, whether they are published or not. The documents may come from teaching and research institutions in France or abroad, or from public or private research centers.
L'archive ouverte pluridisciplinaire $\mathbf{H A L}$, est destinée au dépôt et à la diffusion de documents scientifiques de niveau recherche, publiés ou non, émanant des établissements d'enseignement et de recherche français ou étrangers, des laboratoires publics ou privés.

\section{(이) $\$$}

Distributed under a Creative Commons Attribution - NonCommercial - NoDerivatives $\mid 4.0$ 
Lentiviral expression of rabies virus glycoprotein in the rat hippocampus strengthens synaptic plasticity

Soheil Ghassemi ${ }^{1}$, Tara Asgari ${ }^{1}$, Hadi MirzapourDelavar ${ }^{1}$, Shayan Aliakbari ${ }^{1}$, Hamid Gholami Pourbadie $^{1 *}$, Christophe Prehaud ${ }^{2}$, Monique Lafon ${ }^{2}$, Alireza Gholami ${ }^{3}$, Kayhan Azadmanesh ${ }^{4}$, Nima Naderi $^{5}$, Mohammad Sayyah ${ }^{1 *}$

${ }^{1}$ Department of Physiology and Pharmacology, Pasteur Institute of Iran, Tehran, Iran

${ }^{2}$ Institut Pasteur, Unité de Neuroimmunologie Virale, UMR 3569, CNRS, Paris, France

${ }^{3}$ WHO Collaborating Center for Reference and Research on Rabies, Pasteur Institute of Iran, Tehran, Iran

${ }^{4}$ Department of Virology, Pasteur Institute of Iran, Tehran, Iran.

${ }^{5}$ Department of Pharmacology and Toxicology, School of Pharmacy, Shahid Beheshti University of Medical Sciences, Tehran, Iran

\section{*Corresponding authors:}

Mohammad Sayyah

Department of Physiology and Pharmacology

Pasteur Institute of Iran, Tehran, Iran

Telephone: +98 2164112834

E-mail: sayyahm2@pasteur.ac.ir

ORCID ID: (iD 0000-0003-0603-2444

\section{Hamid Gholami Pourbadie}

Department of Physiology and Pharmacology

Pasteur Institute of Iran, Tehran, Iran

Telephone: +98 2164112834

E-mail: $\underline{\text { h_gholamipour@pasteur.ac.ir }}$

ORCID ID: 0000-0002-7634-7428 


\section{Abstract}

Rabies virus (RABV) is a neurotropic virus exclusively infecting neurons in the central nervous system. RABV encodes five proteins. Among them, the viral glycoprotein (RVG) plays a key role in viral entry into neurons and rabies pathogenesis. It was shown that the nature of the $\mathrm{C}$-terminus of the RABV G protein, which possesses a PDZ binding motif (PBM) modulates the virulence of the RABV strain. The neuronal protein partners recruited by this PBM may alter host cell function. This study was conducted to investigate the effect of RVG on synaptic function in the hippocampal dentate gyrus (DG) of rat.

Two $\mu 1\left(10^{8}\right.$ T.U. $\left./ \mathrm{ml}\right)$ of the lentiviral vector containing RVG gene was injected into the DG of rat hippocampus. After 2 weeks, the rat's brain was cross-sectioned and RVG-expressing cells were detected by fluorescent microscopy. Hippocampal synaptic activity of the infected rats was then examined by recording the local field potentials from DG after stimulation of the perforant pathway. Short term synaptic plasticity was also assessed by double pulse stimulation.

Expression of RVG in DG increased long term potentiation population spikes (LTP-PS), whereas no facilitation of LTP-PS was found in neurons expressing $\triangle R V G$ (deleted PBM). Furthermore, RVG and $\triangle R V G$ strengthened paired pulse facilitation.

Our data demonstrate that RVG expression facilitates both short and long term synaptic plasticity in the DG indicating that it may involve both pre- and post-synaptic mechanisms to alter synaptic function. Further studies are needed to elucidate the underlying mechanisms.

Keywords: Long term potentiation, Dentate gyrus, PDZ binding motif, Plasticity

\section{Introduction}

Rabies is still claiming 50,000 lives per year (Jackson 2010). The etiological agent, rabies virus (RABV), is a neurotropic virus affecting the central nervous system (CNS) of mammalian species. In spite of drastic behavioral changes, mild pathological alterations are usually seen in the rabies-infected brain. This phenomenon makes it difficult to understand the underlying mechanism of extreme behavioral amendments induced by rabies. The poor understanding of the pathogenic mechanisms of RABV may contribute to the failure of new treatment in infected patients. RABV encodes five structural proteins; glycoprotein, phosphoprotein, nucleoprotein, matrix protein, and RNA-dependent RNA polymerase (Tordo and Kouknetzoff 1993). RABV glycoprotein (RVG) is a trimeric type I trans-membrane protein and surfaceexposed viral coat protein with an important role in the rabies pathogenesis by enabling the virus to bind to the receptors at the site of inoculation, to enter into neurons, and to fuse with the endosomal membrane. RVG is also involved in the viral gene transcription/translation, and retrograde transport of the virus to upper neuronal cells (Cherian et al. 5015). Maintenance of the viability of RABV-infected cells is vital for the viral cycle and pathogenesis, yet the molecular mechanisms largely uncharacterized. There are some evidences indicating that incorporation of RVG into the membrane of target cells ascertains the fate of neuronal survival or death (Caillet-Saguy et al. 2015; Artola and Singer 1993).

The death of rabid patients with minor histological sign in their brains highlights the hypothesis that RABV may disrupt the function of nerve cells rather than destroy neurons (Bouzamondo et al. 1993; Tsiang 1993). Preservation of infected neurons is vital for viral dissemination (Lafon 2011). However, hijacking neurons through multiple signaling pathways (Azimzadeh Jamalkandi et al. 2016) may be reflected by aberrant synaptic transmission (Fu and Jackson 2005) or ion channels dysfunctions (Iwata et al. 1999) leading to rabies- 
associated clinical manifestations and ultimately death. Various RABV strains differently affect the homeostasis of infected neurons. Pathogenic or virulent RABV strains survive the host cells, maintain the cell integrity and therefore guarantee viral propagation (Lafon 2011; Artola and Singer 1993). On the other hand, attenuated strains cause neuronal apoptosis, which precludes viral dissemination (Prehaud et al. 2003). It was shown that the difference in the cytoplasmic domain of RVG causes this dual action. The RVG cytoplasmic domain possesses a PDZ-binding motif (PBM). PBM is a protein-protein interaction domain typically recognized by proteins containing PDZ domains and plays a critical role in neuronal homeostasis. RABV may manipulate signaling pathway(s) involved in neuronal physiology through interaction with the PDZ domain of the neuronal microtubule associated serine/threonine kinase 2 (MAST2). Meanwhile, it is reported that the extracellular domain of RVG can also inhibit neurotransmitter receptors thereby change the function of neurons (Lee and Zheng 2010). Yet, data upon the effect of RABV on the function of ion channels and synaptic transmission are limited. RABV has been shown to reduce voltage dependent sodium and inward rectifier potassium channel currents in infected rodent neural cell lines (Iwata et al. 1999; Iwata et al. 2000). It has been reported that release of neurotransmitters such as serotonin, dopamine and norepinephrine is increased in the hippocampal region of the RABV-infected mice at the early stage of the disease (Fu and Jackson 2005). Infecting different cerebral regions, RABV elicits diverse behavioral changes from aggression to hallucination. Given that behavior is a reflection of changes in neuronal/synaptic activity in a specific neural circuit, exploring synaptic response to RABV or RVG will help to further reveal pathogenicity of rabies.

Hippocampus possesses a unique structure, which makes it ideal to study the synaptic function. Longterm potentiation (LTP) is one of the main cellular mechanisms of the hippocampus in dealing with new harmful or pleasant experiences. LTP is an experimental model of synaptic plasticity and an activity-dependent long-term strengthening of excitatory synaptic transmission following applying a transient train of highfrequency electrical stimulation in various cerebral regions including hippocampus (Bliss and Collingridge 1993; Fourcaudot et al. 2009).

Thus, the present study aimed to examine function of rat hippocampal synapses following infection with RVG.

\section{Materials and methods}

Lentiviral vector production

The lentivectors were prepared according to the previously established method (Farzaneh et al. 2018). Briefly, HEK 293T cells (from Cell Bank of Pasteur Institute) were grown in Dulbecco's Modified Eagle Medium (DMEM) supplemented with $10 \% \mathrm{FBS}$ and $100 \mathrm{units} / \mathrm{ml}$ penicillin/streptomycin at $37^{\circ} \mathrm{C}$ in a humidified $5 \%$ $\mathrm{CO} 2$ environment. Plasmids containing full-length RVG of challenge virus standard CVS (G-CVS) or $\triangle \mathrm{RVG}$, in which four amino acids in the PBM site are deleted (Fig. 1) (Khan et al. 2019), were kindly provided by Christophe Prehaud (Neuroimmunologie Virale, Institute Pasteur, Paris, France) and sub-cloned in Plenti7.3 which contains eGFP (Invitrogen, France). The HEK 293T cells were transfected with transfer (p-Lenti-GCVS), envelope (G protein of VSV) and packaging (pMDLg, pRRE and pRSV/Rev) plasmids using traditional calcium phosphate method. Forty eight hours after transfection, the medium was collected and filtered using a $0.45-\mu \mathrm{m}$ filter (Macherey-Nagel, Germany). The medium was then centrifuged at 50,000 rpm for $2 \mathrm{~h}$ at $4^{\circ} \mathrm{C}$ and the viral pellet was resuspended in PBS-BSA $1 \%$ solution and stored at $-80^{\circ} \mathrm{C}$ until use.

\section{Determination of viral titration}


Lentivirus titer was determined using fluorescent microscopy. Briefly, $1 \times 10^{4}$ HEK 293T cells/well were seeded in a 96-well plate. Twenty h later, they were infected by gradient dilution of the $10 \mu 1$ lentiviral particles $\left(1: 10,1: 100,1: 1000,1: 10^{4}, 1: 10^{5}\right.$ and $\left.1: 10^{6}\right)$ in $100 \mu$ final volume of culture medium. Forty eight hours later, fluorescent positive cells were counted using fluorescent microscopy in triplicates and the number of positive cells was determined. Then, the lentivirus titer was estimated using the following formulation: Viral titer $(\mathrm{T} . \mathrm{U} . / \mathrm{ml})=$ number of fluorescent positive cells $\times 10 \times$ dilution.

\section{Animals}

Adult male Wistar rats (220-250g, Pasteur Institute, $\mathrm{n}=70)$ were used. They were housed individually in pairs in plexiglass cages with free access to food and water in a $12 \mathrm{~h}$ light/12h-dark cycle. All efforts were made to use minimal number of rats and also diminish animal suffering during the study. Experiments were carried out in accordance with the Review Board and Ethics Committee of Pasteur Institute (Authorization code 93-0201785, 22 April 2014) and conform to the European Communities Council Directive 2010-63-EU.

\section{Surgical procedures}

Rats were anesthetized with intraperitoneal (i.p.) injection of ketamine $(100 \mathrm{mg} / \mathrm{kg})$ and xylazine $(10 \mathrm{mg} / \mathrm{kg})$. Then, $2 \mu \mathrm{l}\left(10^{8}\right.$ T.U. / ml) of RVG, or $\triangle \mathrm{RVG}$ lentivirus, or phosphate buffer solution (PBS as control) were injected by stereotaxic surgery into the dentate gyrus $(\mathrm{DG} ; \mathrm{AP}=-3.8, \mathrm{ML}=2.4, \mathrm{DV}=-3.5)$ using a Hamilton microsyringe.

\section{Histology}

Two weeks after injection of the lentivector, brain of the rats (3 rats in each group) was dissected out for histological examinations. The animals were euthanized by $\mathrm{CO} 2$ asphyxiation and perfused transcardially with $4 \%$ paraformaldehyde in $0.1 \mathrm{M}$ PBS. The brains were harvested, kept in $10 \%$ paraformaldehyde in $0.1 \mathrm{M}$ PBS and processed for embedding in paraffin blocks. The brains were then cut horizontally to $8 \mu \mathrm{m}$ in thickness. The sections were deparaffinaized, rehydrated in a descending alcohol series and stained with 4',6-diamidino2-phenylindole (DAPI, 100ng/m in tris buffered saline, Sigma), cover slipped with $90 \%$ glycerol mounting buffer and visualized in the dark place with fluorescent microscope (Nikon, Japan) equipped with specific filter cube for FITC fluorescence channels, and connected to a digital camera. Digital photographs were taken using 10x objective lenses.

\section{Electrophysiological recordings}

Two weeks after lentivector injection into DG, animals were anaesthetized by urethane $(1.5 \mathrm{~g} / \mathrm{kg})$ and fixed in a stereotaxic apparatus. A bipolar stimulating electrode, which was a twisted-pair stainless steel wires (A-M Systems, USA) was placed at the perforant path (AP: 8.1, ML: 4.3, DV: 3.3), and an analogous electrode was placed at DG (AP: 3.8, ML: 2.4, DV: 3-3.5) as a recording electrode. Both electrodes were gently lowered and the pathway was stimulated until a standard synaptic signal was obtained. After a 30-min stabilization period, the recording protocol was started. Field potentials were evoked by a $0.2 \mathrm{~ms}$ monophasic square current using a stimulator/isolator (A365, WPI, USA), they were then amplified and filtered (between $1 \mathrm{~Hz}$ and $3 \mathrm{kHz}$ ) using a differential amplifier (DAM 80, WPI, USA) and digitized (10 kHz) and analyzed by a homemade digitizer/software. The amplitude between the first positive upsurge and the most negative point was measured as the PS amplitude. Input/output (I/O) curve of PS amplitude was plotted against different stimulating current magnitudes. A test stimulus with the intensity of $50 \%$ of the maximum response obtained from I/O curve was 
applied every $5 \mathrm{~min}$ for $30 \mathrm{~min}$ to record baseline synaptic activity. A high-frequency stimulation (HFS), consisting of 10 trains of 20 pulses at $400 \mathrm{~Hz}$ with $80 \%$ of maximum intensity delivered every $10 \mathrm{~s}$, was used to induce LTP. The criteria for LTP induction was at least $25 \%$ increase of the PS amplitude compared to the baseline. After applying HFS, the synaptic responses were recorded every $5 \mathrm{~min}$ for $90 \mathrm{~min}$. The paired field potentials were also recorded. They were elicited by paired pulses of stimuli with interpulse intervals (IPIs) ranging from 25 to $370 \mathrm{~ms}$. The paired-pulse ratio (PPR) was the result of dividing PS amplitude of the second field potential by that of the first one.

For LTD recording, in order to induce LTD in the DG both medaial (MPP, AP: $-7.9 \mathrm{~mm}, \mathrm{~L}:+4.2 \mathrm{~mm}$, DV= $2.5 \mathrm{~mm}$ ) and lateral (LPP, AP: $-8.0 \mathrm{~mm}, \mathrm{~L}:+5.0 \mathrm{~mm}, \mathrm{DV}:-2.8 \mathrm{~mm}$ ) perforant pathways are implanted with teflon-coated, stainless steel recording electrodes(ref) and recording electrode is situated in the hilar region of the DG. A test stimulus with the intensity of $50 \%$ of the maximum response obtained from I/O curve was applied every $2 \mathrm{~min}$ for $14 \mathrm{~min}$ to record baseline synaptic activity. The MPP was stimulated by eight stimulus trains of $2 \mathrm{~s}$ duration, spaced 1 minute apart. Within each train, the stimuli occurred as a burst of 5 pulses at $100 \mathrm{~Hz}$, repeated at $200 \mathrm{~ms}$ intervals. The lateral path, which served as the test pathway was stimulated by single pulses (low frequency stimulation), equally spaced between the MPP bursts.

\section{Statistical analysis}

The electrophysiology experiments were repeated 3 times $(n=7-10)$, each time by a different expert experimenter who was unaware of the nature of the treatment. The data are expressed as means \pm S.E.M. Oneway or two-way analysis of variance and Post-hoc Tukey's test were used for multiple comparisons. In all cases, a $p \leq 0.05$ was considered statistically significant.

\section{Results}

\section{RVG expression in the hippocampus}

The titer of the lentivector was found $2 \times 10^{8}$ T.U. /ml. Fig. 2 A shows that RVG/eGFP gene was successfully expressed in granule cells two week after delivery of the lentivector into DG. The nuclear DNA was stained with DAPI (Fig. 2B). Merge picture indicates the co-localization between the nucleus and GFP proteins (Fig. 2C). In the injection side (supra-pyramidal blade of DG) over $90 \%$ of the counted DG granule cells expressed bright green fluorescence.

\section{RVG expression in the hippocampal DG facilitated short- and long-term synaptic plasticity}

Synaptic activity was recorded 30 min before applying HFS. Test stimuli were determined from intake output (I/O) curve of PS. PS amplitudes was obtained in DG following stimulation of the perforant pathway with different intensities (Fig 3A). They were similar for each experimental condition. On the contrary, basal synaptic responses in the DG in response to perforant pathway stimulation with $50 \%$ maximum intensity (treatment factor; $\mathrm{F}(2,168)=34.03, \mathrm{p}<0.0001)$ were greater ( $>$ two fold) in RVG $(\mathrm{n}=10)$ group compared to the control $(\mathrm{n}=11, \mathrm{p}<0.05)$ and $\Delta \mathrm{RVG}(\mathrm{n}=10, \mathrm{p}<0.05)$ groups. No difference was found between control and $\triangle \mathrm{RVG}$ groups (Fig $3 \mathrm{~B}$ ). 
To determine whether the enhanced synaptic transmission observed in RVG group was attributable to presynaptic or postsynaptic mechanisms, we measured paired-pulse facilitation in the DG following double stimulation of perforant pathway with different inter pulse intervals. In paired pulse stimulation, second stimulation of equal intensity normally evokes a greater synaptic response than the first and is used to determine probability of presynaptic transmitter release. Fig. 4A shows representative paired field potential recordings with $75 \mathrm{~ms}$ inter-event interval. As shown in Fig. 4B and C, at 25 inter-pulse intervals (IPI), synaptic responses in control group ( $n=4)$ display paired-pulse depression whereas in RVG $(n=8)$ and $\Delta R V G(n=7)$ groups paired pulse facilitation occurred. Also, synaptic facilitation in other IPIs was more prominent in RVG and $\triangle \mathrm{RVG}$ compared to the corresponding synaptic response for control group, indicating that RVG facilitates release probability of excitatory neurotransmitter independent to its PBM site.

Post synaptic changes were investigated by extracellular field potential recording. Fig. 5A shows representative field potential recordings at baseline and after induction of LTP (post HFS) at different time points. The time course of PS-LTP induction in the experimental groups is shown in Fig. 5B. Two-way ANOVA revealed a significant effect of treatment $[\mathrm{F}(1,270)=61.59$, $\mathrm{P}<0.0001$, Fig. 5B]. RVG expression $(n=8)$ in the hippocampal DG augmented PS-LTP amplitude following stimulation of perforant pathway in all time points compared to the control group. However, no difference of PS-LTP amplitude was found between control and $\triangle \mathrm{RVG}$ groups indicating that deletion of the PBM motif blocked the facilitating effect of RVG on synaptic plasticity (Fig. 5B). Fig. 5C demonstrates the PS amplitudes in time point of 10, 45 and 90 min in different experimental groups demonstrating that RVG expression led to a significant increase of PS amplitude compared to the control and $\triangle \mathrm{RVG}$ groups ( $\mathrm{n}=7$ and 10 , respectively). Overall, these data indicate that unlike presynaptic function, postsynaptic alteration due to RVG expression in DG is dependent to its PBM sequence.

\section{RVG blocked synaptic long term depression in the DG}

Fig. 6 A shows the associative paradigm of long term depression (LTD) induction in which both MPP (with HFS) and LPP (with HFS) were concurrently stimulated (fig. 6 A). Two-way ANOVA showed a significant effect of treatment $F(2,352)=18.19, p<0.0001$, Fig. 5B].The paradigm induced 15-20\% decrease of PS amplitude in the control group. However, expression of RVG in the DG suppressed LTD induction. No significant difference of PS amplitude was found between control $(n=8)$ and RVG $(n=9)$ groups until 20 min time point, however, from the time point of $20 \mathrm{~min}$ after LTD induction the difference increased and reached to significant levels $(\mathrm{p}<0.05)$. There was no significant difference of PS amplitude between control and $\triangle \mathrm{RVG}$ groups indicating that RVG dependent to its PBM motif may block long-term synaptic suppression (Fig. 6B). Fig. $6 \mathrm{C}$ and D demonstrate the PS amplitudes in time point of 10 and 28 min time points, respectively. No difference was found among groups at the time point of $10 \mathrm{~min}$, but RVG significantly increased PS amplitude (135 $\pm 14 \%$ of baseline) at the 28 min time point. Deletion of PBM site in $\Delta$ RVG group resulted in significant decrease of PS amplitude ( $88.7 \pm 3.7 \%$ of baseline) at the time point of $28 \mathrm{~min}$ compared to the RVG group.

\section{Discussion}


The current study provides evidence for interplay between RVG and synaptic function. We found that induction of RVG expression facilitates basic excitatory synaptic transmission and LTP expression in the hippocampal DG through a PBM dependent manner. Unlike $\triangle$ RVG, RVG blocked LTD in the DG. Furthermore, we found also that RVG strengthens paired pulse facilitation in DG in all inter-pulse intervals. This data indicate that RVG may involve both pre- and post- synaptic elements through different mechanisms to alter synaptic function.

RVG which plays a predominant role in the pathogenesis of rabies is a type I glycoprotein which constitutes the trimeric spikes of the virus particle. RVG interacts with cellular receptors, embeds in lipid membrane of the host cell, mediates $\mathrm{pH}$-dependent fusion, and promotes trans-synaptic spread within the CNS (Yousaf et al. 2012). While rabies causes extreme behavioral and neurological disturbances with a $100 \%$ fatality rate in humans, relatively mild pathological changes are observed in the infected brain (Fu and Jackson 2005). The underlying mechanism inducing such furious behavioral changes remains poorly understood. Several hypotheses including abnormal synaptic transmission involving serotonin (Bouzamondo et al. 1993), acetylcholine (Dumrongphol et al. 1996), GABA (Ladogana et al. 1994) and dopamine (Fu and Jackson 2005), electrophysiological changes (Gourmelon et al. 1991) and ion channel dysfunction in voltage gated $\mathrm{Na}^{+}$and inward rectifier K+ channels (Iwata et al. 1999), or disrupted RNA and protein synthesis (Gosztonyi 1994) have been reported for RABV pathogenesis. Furthermore, myeloid differentiation primary response protein (MyD88) dependent pathway (Azimzadeh Jamalkandi et al. 2016) and MAST2/ phosphatase and tensin homolog (PTEN) signaling pathway (Caillet-Saguy et al. 2015) have been suggested to be involved in the rabies pathogenesis. Also, the virus may induce neuronal dysfunction through acting on fundamental neuronal elements including ion channels to provoke the clinical manifestation rather than neurodegeneration (Lee and Zheng 2010). Although rabies pathogenicity is a multigenic trait, the effect of RABV on the brain function could be attributed to its RVG as a major contributor to the RABV pathogenicity (Pulmanausahakul et al. 2008; Caillet-Saguy et al. 2015; Khan et al. 2019; Lafon 2011). It has been reported that ion channels could be an important target for viruses to alter cellular function (Mankouri et al. 2009). In this line, RABV may also alter neuronal and ultimately cerebral function through action on ion channels. Iwata et al. showed that RABV inhibited voltage-dependent sodium and inward rectifier potassium channels and changed the resting membrane potential resulting in membrane depolarization (Iwata et al. 1999). Street RABV has been shown to induce progressive disappearance of the all stages of sleep waves in the infected mice and increases the duration of waking stages prior to clinical manifestation (Gourmelon et al. 1991) indicating a modulation of neuronal excitability in specific cerebral regions responsible for sleep-waking phases. Furthermore, it has been reported that following infection of rats with RABV, release of neurotransmitters in the hippocampus elevated on the first day, reached to a peak on the third day, and then declined on the fifth day (Fu and Jackson 2005). Progressive neurotransmitter release after RABV inoculation could lead to facilitate synaptic transmission and ultimately modulate neuronal excitability which may contribute to clinical symptoms of agitation, hydrophobia, wild aggressiveness, and even seizures in the infected subjects (Hemachudha et al. 1989).

Distribution of the RABV antigen in various cerebral areas is well documented in diverse species, in which hippocampus has been an ideal region for RABV detection (Stein et al. 2010; Song et al. 2013). In current study, hippocampus was targeted to examine the effect of RVG on synaptic function. Experiencebased alteration in synaptic strength underlies memory formation. These changes include persistent long- LTP or LTD of synaptic transmission. It has been believed that LTD along with LTP underlies storage of memory (Bear 1996). We found that induction of RVG expression in the hippocampus leads to enhanced synaptic transmission. This synaptic facilitation may be due to increased neurotransmission release from the pre- 
synaptic terminals, alteration of the post-synaptic membrane, or both. We found in the present study that RVG could induce both short and long term synaptic potentiation. Therefore, it seems that both presynaptic and postsynaptic elements are modulated by RABV. We found that the facilitation on LTP was absent when the C terminal PBM site was missing, indicating that RVG may alter synaptic function in the host cells through its PBM region. We also demonstrate that RVG dependent to its BM site blocked LTD in the DG. Both LTP and LTD are the cellular molecular mechanism of memory formation. Unlike LTP, LTD is most reliably elicited by elongated low frequency stimulation, which evoked non-significant postsynaptic depolarization. It is believed that the magnitude of postsynaptic depolarization and resultant increase of intracellular $\mathrm{Ca}^{2+}$ concentration determine whether the synapse undergoes LTP or LTD (Artola and Singer 1993; Cummings et al. 1996). Here, we found that animals expressing RVG in the DG express LTP instead of LTD, however, deleting PBM motif restored LTD expression. RVG may alter depolarization state of postsynaptic cells so that they show LTP in response to associative stimulation of LPP and MPP, which normally elicits LTD in the DG. In support to this view, it has been reported that depending on the postsynaptic depolarization level in CA1 cells, four train of a $5 \mathrm{~Hz}, 2 \mathrm{~s}$ stimulus elicit either LTD or LTP (Stanton and Sejnowski 1989). Furthermore, tetanic stimulation has also been reported to elicit NMDA related LTP or LTD in DG depending on the level of postsynaptic depolarization during the tetanus (Xie et al. 1992). Further study using intracellular recording is required to elucidate how RVG change membrane potential or synaptic inputs (IPSCs and EPSCs) in the DG.

We found that RVG facilitated short-term synaptic transmission (paired pulse facilitation) indicating an increase probability of neurotransmitter release from pre-synaptic terminals. This effect of RVG was independent of its PBM site. Consistent with this finding, it has been shown that RABV-infected NG108-15 cells displayed decreased response to $\alpha 2$-adrenoreceptor agonists (Iwata et al. 2000). It is believed that $\alpha 2$ adrenoreceptor serves as a brake to keep neurons from releasing their neurotransmitters beyond physiological needs through blockade of voltage-dependent calcium channels (Iwata et al. 2000). The altered modulation of voltage dependent calcium channels may underlie facilitated synaptic transmission due to RVG observed in our study. Fu and Jackson reported that the release of serotonin, norepinephrine and dopamine was increased in the hippocampus of rat following intranasal inoculation with RABV CVS-24 (Fu and Jackson 2005). On the other hand, it has been shown that the two important synaptic proteins, tripartite motif-containing protein 9 (TRIM9) and $\alpha$-soluble NSF-attachment protein ( $\alpha$-SNAP), were down-regulated in silver-haired bat rabies virus-infected mice whereas infection with attenuated RABV strain (CVS-B2C) did not change the expression level of these two synaptic proteins (Dhingra et al. 2007). In other words, pathogenic RABV strains may blunt synaptic function through downregulation of critical synaptic proteins. However, it should be noted that these proteins were measured in these studies after development of severe paralysis in mice. It may seem that neurotransmitter release is stimulated by the virus in the early stage of the infection but at later stages of infection, neurons are no longer capable of releasing neurotransmitters at the synaptic clefts. Decreased neurotransmitter release in the late stage of disease may be also due to membrane depolarization beyond threshold which results in inactivation of voltage dependent $\mathrm{Na}^{+}$and $\mathrm{Ca}^{2+}$ channels and blunt neuronal activation and synaptic transmission. Decreasing neurotransmitter release may also be due to microstructural changes in the infected neurons at the late stage of the disease. In this line, Scott et al., found relatively few changes in the perikarya and neuronal processes in the hippocampi of moribund CVS- infected YFP mice (Scott et al. 2008). Furthermore, disorganized apical dendrites of neurons have been reported in the hippocampus of mice infected with a pathogenic RABV strain, CVS-N2C (Li et al. 2005). It was also shown that RABV did not inhibit gene and protein synthesis in cell culture (Ermine and Flamand 1977; Madore and 
England 1977) and RABV failed to shut down host cellular DNA, RNA, or protein synthesis in infected brain because of its limited or late onset of cytopathogenic effect (Baer et al. 1990).

RVG may also act through postsynaptic elements to induce synaptic facilitation. In most cases, LTP is dependent on molecular changes including increased AMPA trafficking and NMDA receptors at the postsynaptic membrane. These receptors at the glutamatergic excitatory synapses are accumulated by scaffolding proteins including membrane-associated guanylate kinases (MAGUKs) at the post-synaptic density (PSD). Synaptic incorporation of AMPA receptors containing GluR1 subunit is involved in LTP induction (Hayashi et al. 2000; Shi et al. 2001). Increased GluR1 in the expression site of RVG may secondarily be due to increased neuronal or synaptic activity in response to RVG. In support of this view, recent studies using tracking of individual AMPA receptors showed that the increased synaptic activity could recruit them at synapses (Opazo and Choquet 2011). Interestingly, Khan and his colleagues have recently reported that Neurovita, a C terminal RVG-derived peptide protects neuronal homeostasis and stimulates neurite outgrowth in several neuron types and the effect was dependent to the PBM site (Khan et al. 2019). In current study, we found that induction of RVG expression in the dorsal hippocampal DG led to facilitate synaptic activity in a PBM dependent manner. While no specific electrophysiological study has tested the effect of RABV on synaptic transmission, some evidence indicates that the virus alters neurotransmitter releases in various brain regions, which can be mirrored in multiple clinical manifestations. For instance, hyperexcitability and aggression may be due to increased synaptic activity in the relevant centers including limbic system (amygdala and hypothalamus), or hallucination may be resulted from altered synaptic transmission in the cerebral cortices. Increased activity of areas involved in the autonomic system, such as the hypothalamus, leads to hyper-salivation and hyper-ejaculation in rabid patients (Tian et al. 2019). Furthermore, increased neuronal activity at vital centers in the brainstem, such as the respiratory control center, can functionally alter the pattern of breathing from ramping to the explosive discharge of neurons, hiccups (DelRosso and Hoque 2013), and eventually hypopnea and apnea. Interestingly, the positive role of DG in the control of fear and anxiety has been widely reported by animal and human studies (Cummings et al. 1996; Stanton and Sejnowski 1989; Xie et al. 1992). Our finding on the increased synaptic activity of DG neurons upon infection by RVG might be a physiologic response to counterbalance the fear and anxiety, the typical feature of patients with rabies. Nonetheless, more and specific studies are needed to confirm this suggestion.

\section{Conclusion}

To the best of our knowledge, this is the first report indicating that RVG facilitates hippocampal short and long term synaptic strength. Short synaptic plasticity is determined by paired pulse facilitation and further study may need to confirm this result. Overall, our findings indicate that RABV may functionally alter synaptic transmission in different cerebral regions through, in part, incorporation of its PBM site. This increased synaptic function might underlie the neurobehavioral disorders seen in rabies disease, or on the other hand be a reaction to correct the disturbance of the nervous system induced by the rabies virus. Comprehensive studies are required to elucidate these assumptions and the mechanisms involved in impact of RABV/RVG on synaptic function.

\section{Compliance with Ethical Standards}

\section{Conflict of interest}

The authors have no conflict of interest to declare.

\section{Ethical approval}


All animal experiments were carried out in accordance with the Review Board and Ethics Committee of Pasteur Institute (Authorization code 93-0201-785, 22 April 2014) and conform to the European Communities

Council Directive 2010-63-EU.

Informed consent

Not applicable.

\section{References}

Artola A, Singer W (1993) Long-term depression of excitatory synaptic transmission and its relationship to long-term potentiation. Trends in neurosciences 16 (11):480-487

Azimzadeh Jamalkandi S, Mozhgani S-H, Gholami Pourbadie H, Mirzaie M, Noorbakhsh F ,Vaziri B, Gholami A, Ansari-Pour N, Jafari M (2016) Systems biomedicine of rabies delineates the affected signaling pathways. Frontiers in microbiology 7:1688

Baer G, Bellini W, Fishbein D (1990) Rhabdoviruses. In" Virology"(B. N. Fields and DM Knipe Eds .883, ( Raven Press, Ltd., New York,

Bear MF (1996) A synaptic basis for memory storage in the cerebral cortex. Proceedings of the National Academy of Sciences 93 (24):13453-13459

Bliss TV, Collingridge GL (1993) A synaptic model of memory: long-term potentiation in the hippocampus. Nature 361 (6407):31

Bouzamondo E, Ladogana A, Tsiang H (1993) Alteration of potassium-evoked 5-HT release from virusinfected rat cortical synaptosomes. Neuroreport 4 (5):555-558

Caillet-Saguy C, Maisonneuve P, Delhommel F, Terrien E, Babault N, Lafon M, Cordier F, Wolff N (2015) Strategies to interfere with PDZ-mediated interactions in neurons: what we can learn from the rabies virus. Progress in biophysics and molecular biology 119 (1):53-59

Cherian S, Singh R, Anjaneya, KP S (5015) Rabies Glycoprotein: A Benefit to the Virus, us or both? Journal of Veterinary Sciences 1 (1):73-81

Cummings JA, Mulkey RM, Nicoll RA, Malenka RC (1996) Ca2+ signaling requirements for long-term depression in the hippocampus. Neuron 16 (4):825-833

DelRosso L, Hoque R (2013) A case of obstructive sleep apnea, gastroesophageal reflux disease, and chronic hiccups: will CPAP help? Journal of Clinical Sleep Medicine 9 (01):92-95

Dhingra V, Li X, Liu Y, Fu ZF (2007) Proteomic profiling reveals that rabies virus infection results in differential expression of host proteins involved in ion homeostasis and synaptic physiology in the central nervous system. Journal of neurovirology 13 (2):107-117

Dumrongphol H, Srikiatkhachorn A, Hemachudha T, Kotchabhakdi N, Govitrapong P (1996) Alteration of muscarinic acetylcholine receptors in rabies viral-infected dog brains. Journal of the neurological sciences 137 (1):1-6

Ermine A, Flamand A Rna syntheses in BHK21 cells infected by rabies virus. In: Annales de microbiologie, 1977. vol 4. pp 477-488

Farzaneh M, Sayyah M, Eshraghi HR, Panahi N, Delavar HM, Pourbadie HG (2018) Transduction efficacy and retrograde movement of a lentiviral vector pseudotyped by modified rabies glycoprotein throughout the trisynaptic circuit of the rat hippocampus. The journal of gene medicine 20 (9):e3046

Fourcaudot E, Gambino F, Casassus G, Poulain B, Humeau Y, Lüthi A (2009) L-type voltage-dependent Ca 2+ channels mediate expression of presynaptic LTP in amygdala. Nature neuroscience 12 1093:(9)

Fu ZF, Jackson AC (2005) Neuronal dysfunction and death in rabies virus infection. Journal of neurovirology 11 (1):101-106

Gosztonyi G (1994) Reproduction of lyssaviruses: ultrastructural composition of lyssavirus and functional aspects of pathogenesis. Current topics in microbiology and immunology 187:4368

Gourmelon P, Briet D, Clarençon D, Court L, Tsiang H (1991) Sleep alterations in experimental street rabies virus infection occur in the absence of major EEG abnormalities. Brain research-1) 554 165-159:(2 
Hayashi Y, Shi S-H, Esteban JA, Piccini A, Poncer J-C, Malinow R (2000) Driving AMPA receptors into synapses by LTP and CaMKII: requirement for GluR1 and PDZ domain interaction. Science 287 (5461):2262-2267

Hemachudha T, Tirawatnpong $S$,Phanthumchinda K (1989) Seizures as the initial manifestation of paralytic rabies. Journal of neurology, neurosurgery, and psychiatry 52 (6):808

Iwata M, Komori S, Unno T, Minamoto N, Ohashi H (1999) Modification of membrane currents in mouse neuroblastoma cells following infection with rabies virus. British journal of pharmacology 126 (8):1691-1698

Iwata M, Unno T, Minamoto N, Ohashi H, Komori S (2000) Rabies virus infection prevents the modulation by $\alpha 2$-adrenoceptors, but not muscarinic receptors, of $\mathrm{Ca}+2$ channels in NG10815 cells. European journal of pharmacology 404 (1-2):79-88

Jackson AC (2010) Why does the prognosis remain so poor in human rabies? Expert review of antiinfective therapy 8 (6):623-625

Khan Z, Terrien E, Delhommel F, Lefebvre-Omar C ,Bohl D, Vitry S, Bernard C, Ramirez J, Chaffotte A, Ricquier K (2019) Structure-based optimization of a PDZ binding motif within a viral peptide stimulates neurite outgrowth. Journal of Biological Chemistry:jbc. RA119. 008238

Ladogana A, Bouzamondo E, Pocchiari M, Tsiang H (1994) Modification of tritiated $y$-amino-n-butyric acid transport in rabies virus-infected primary cortical cultures. Journal of general virology 75 (3):623-627

Lafon M (2011) Evasive strategies in rabies virus infection. Advances in virus research 79:33-53. doi:10.1016/b978-0-12-387040-7.00003-2

Lee H-J, Zheng JJ (2010) PDZ domains and their binding partners: structure, specificity, and modification. Cell Communication and Signaling 8 (1):8

Li X-Q, Sarmento L, Fu ZF (2005) Degeneration of neuronal processes after infection with pathogenic, but not attenuated, rabies viruses. Journal of virology 79 (15):10063-10068

Madore HP, England JM (1977) Rabies virus protein synthesis in infected BHK-21 cells. Journal of virology $22(1): 102-112$

Mankouri J, Dallas ML, Hughes ME, Griffin SD, Macdonald A, Peers C, Harris M (2009) Suppression of a pro-apoptotic $\mathrm{K}+$ channel as a mechanism for hepatitis $\mathrm{C}$ virus persistence. Proceedings of the National Academy of Sciences 106 (37):15903-15908

Opazo P, Choquet D (2011) A three-step model for the synaptic recruitment of AMPA receptors. Molecular and Cellular Neuroscience 46 (1):1-8

Prehaud C, Lay S, Dietzschold B, Lafon M (2003) Glycoprotein of nonpathogenic rabies viruses is a key determinant of human cell apoptosis. J Virol 77 (19):10537-10547. doi:10.1128/jvi.77.19.10537-10547.2003

Pulmanausahakul R, Li J, Schnell MJ, Dietzschold B (2008) The glycoprotein and the matrix protein of rabies virus affect pathogenicity by regulating viral replication and facilitating cell-to-cell spread. Journal of virology 82 (5):2330-2338

Scott CA, Rossiter JP, Andrew RD, Jackson AC (2008) Structural abnormalities in neurons are sufficient to explain the clinical disease and fatal outcome of experimental rabies in yellow fluorescent protein-expressing transgenic mice. Journal of virology 82 (1):513-521

Shi S-H, Hayashi Y, Esteban JA, Malinow R (2001) Subunit-specific rules governing AMPA receptor trafficking to synapses in hippocampal pyramidal neurons. Cell 105 (3):331-343

Song Y, Hou J, Qiao B, Li Y, Xu Y, Duan M, Guan Z, Zhang M, Sun L (2013) Street rabies virus causes dendritic injury and F-actin depolymerization in the hippocampus. Journal of General Virology $94(2): 276-283$

Stanton PK, Sejnowski TJ (1989) Associative long-term depression in the hippocampus induced by hebbian covariance. Nature 339 (6221):215-218

Stein L, Rech R, Harrison L, Brown C (2010) Immunohistochemical study of rabies virus within the central nervous system of domestic and wildlife species. Veterinary Pathology 47 (4):630-633

Tian Z, Chen Y, Yan W (2019) Clinical features of rabies patients with abnormal sexual behaviors as the presenting manifestations: a case report and literature review. BMC infectious diseases 19 (1):679

Tordo N, Kouknetzoff A (1993) The rabies virus genome: an overview. Onderstepoort J Vet Res 60 (4):263-269 
Tsiang H (1993) Pathophysiology of rabies virus infection of the nervous system. In: Advances in virus research, vol 42. Elsevier, pp 375-412

Xie X, Berger TW, Barrionuevo G (1992) Isolated NMDA receptor-mediated synaptic responses express both LTP and LTD. Journal of neurophysiology 67 (4):1009-1013

Yousaf MZ, Qasim M, Zia S, Ashfaq UA, Khan S (2012) Rabies molecular virology, diagnosis, prevention and treatment. Virology journal 9 (1):1-5

Figures: 


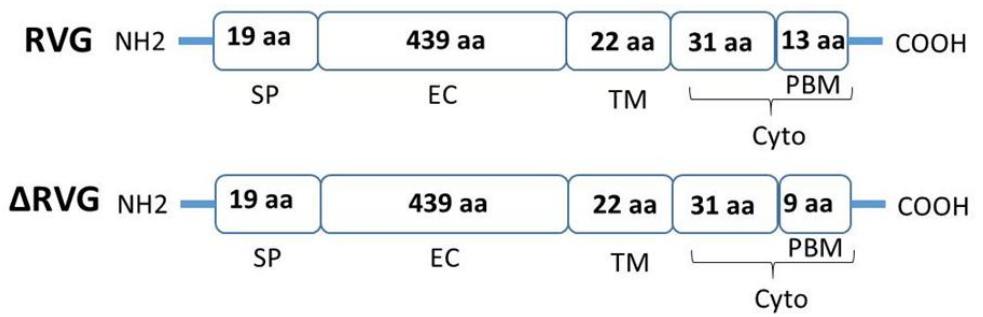

Fig. 1 Structures of the RVG and $\triangle R V G$.
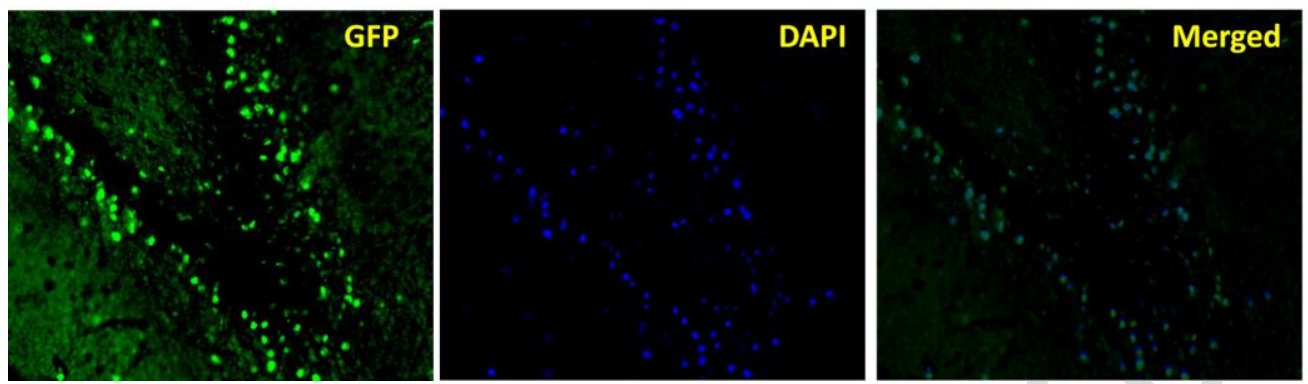

Fig. 2 Analysis of DAPI and GFP-fluorescence distribution in DG granule cells transfected with plasmids expressing eGFP and RVG proteins. A) Green cells indicate the successful expression of the RVG/GFP gene in hippocampal granule cells two weeks after microinjection of the lentivector into the DG. B) The nuclear DNA was stained with DAPI. C) Merge picture was used to confirm the co-localization between the nucleus and GFP protein. (magnification: 100x, scale bar: $50 \mu \mathrm{m}$ ).

A

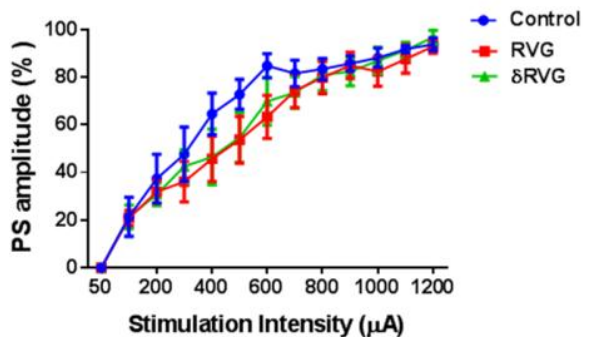

B

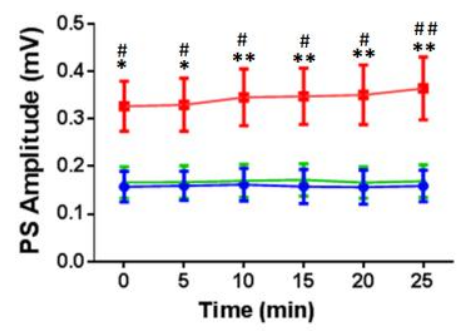

Fig. 3 RVG facilitates basal synaptic transmission in the DG. A) Input and output curve indicates no difference of normalized responses to seven stimulus intensities between groups. B) Baseline responses to the $50 \%$ maximum of intensity during $30 \mathrm{~min}$ are more prominent rats expressing RVG $(\mathrm{n}=10)$ in their hippocampal DG compared to control $(n=11)$ and $\Delta \mathrm{RVG}(\mathrm{n}=10)$ groups. ${ }^{*} \mathrm{p}<0.05$ and. ${ }^{* *} \mathrm{p}<0.01$ compared to the control group. ${ }^{\#} \mathrm{p}<0.05$ and .\# $\mathrm{p}<0.01$ compared $\Delta \mathrm{RVG}$ and $\mathrm{RVG}$ groups. 

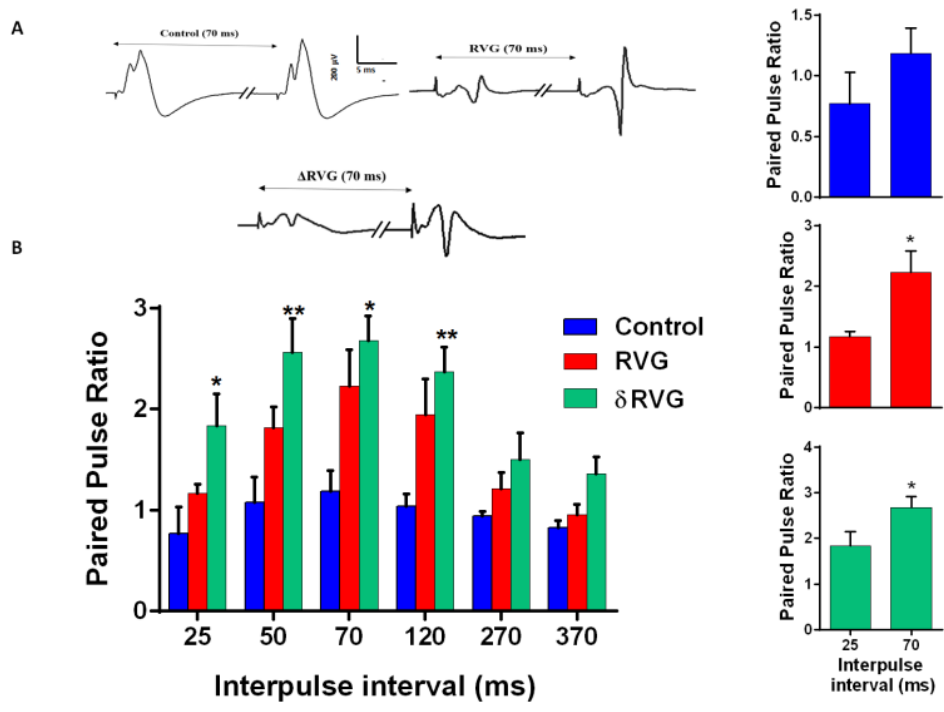

Fig. 4 Induced expression of RVG in the hippocampal DG facilitates paired pulse facilitation. A) Representative field potential responses in the DG evoked by double stimulation of perforant pathway with 70 ms inter-pulse interval (IPI). B) Unlike RVG $(n=8)$ and $\Delta$ RVG $(n=7)$, control group $(n=4)$ showed paired pulse inhibition in the IPI $25 \mathrm{~ms}$. RVG expression in the DG increases the ratio (the relative PS amplitude of the first and second pulse) in all IPIs but fails to reach significant level. Expression of $\triangle R V G$ (with deleted PBM motif) also significantly increases paired pulse facilitation compared to the control group. In the IPI of 50-120 ms the PS amplitude of the second response are more than two time of the first one in the animals expressing $\triangle R V G$ in DG. C) No difference is observed between IPI of 25 and $70 \mathrm{~ms}$ in the control group. However, the paired pulse ration in RVG and $\triangle \mathrm{RVG}$ groups is significantly increased in $70 \mathrm{~ms}$ compared to that of 25 ms. $* p<0.05$ and $* * p<0.05$ compared to the control group.

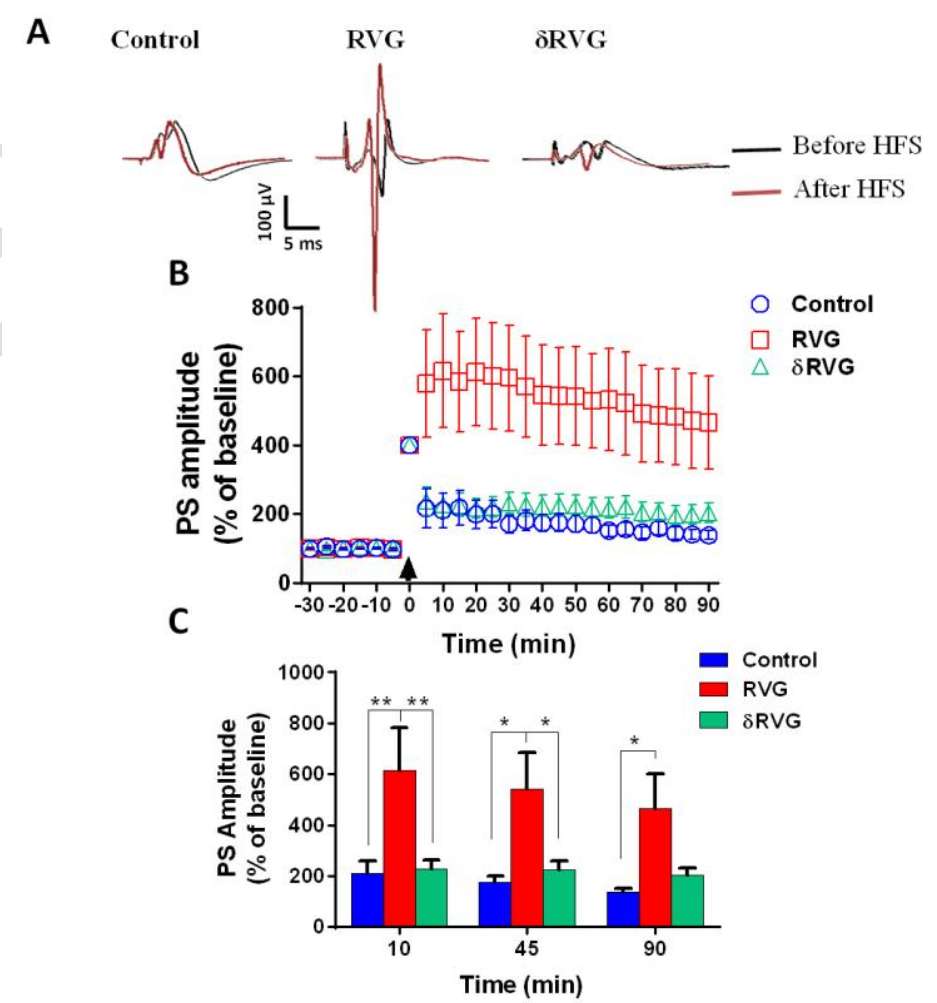


Fig. 5 Induced expression of RVG in the hippocampal DG facilitates LTP expression. A) Representative field potential responses in the DG before and after HFS of the perforant pathway in all experimental groups. B) The time course of PS-LTP induction in experimental groups. RVG expression facilitates LTP induction in all time points compared to the control group. However, deletion of PBM motif in the RVG decreases the synaptic response toward the control level. C) PS-LTP amplitude at 10, 45 and 90 min after applying HFS. Expression of RVG significantly increases PS amplitude in all time points compared to the control group and the synaptic responses are reversed to the control level in the $\triangle R V G$ group. Number of subjects in control, $R V G$ and $\Delta R V G$ groups were 7,8 and 10 , respectively.

A
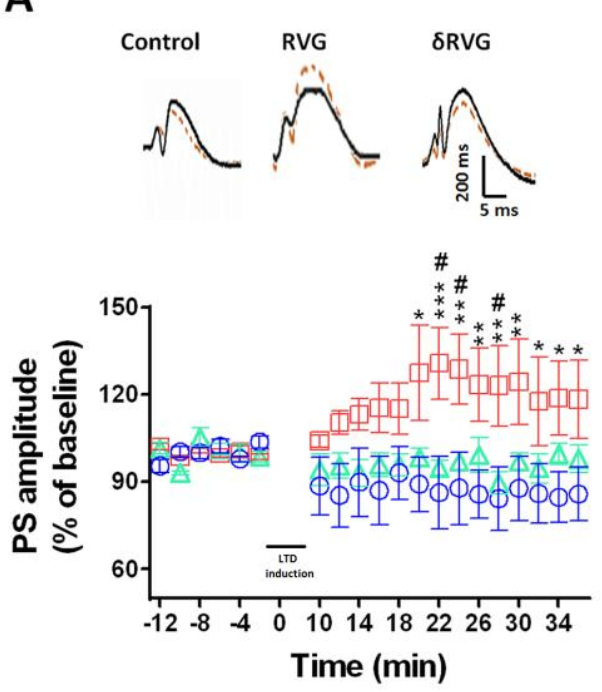

B

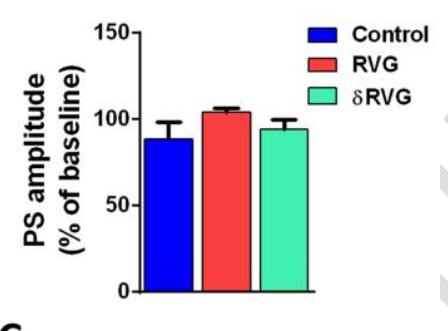

$\square$ RVG

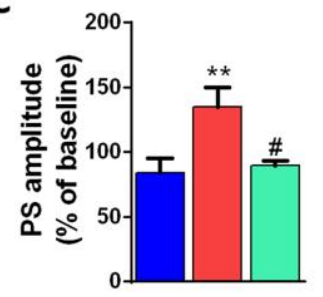

Fig. 6 RVG expression in the hippocampus blocked long term synaptic depression. A) upper panel demonstrates representative field potential responses before and after LTD induction in the DG of all experimental groups. B) Control group expressed LTD, however, RVG expression suppressed LTD induction in the DG at all time points (the difference increased and reached to a significant level after $20 \mathrm{~min}, \mathrm{p}<0.05$, compared to the control group). However, PBM deletion of RVG resulted in LTD expression in the DG of the $\triangle$ RVG group. C and D) PS-LTD amplitude at 10 and $28 \mathrm{~min}$ after LTD. There was no significant difference among groups at the $10 \mathrm{~min}$ time points. However, at the time point of $28 \mathrm{~min}$, PS amplitude was increased in RVG group compared to the control $(\mathrm{p}<0.01)$ and $\Delta \mathrm{RVG}(\mathrm{p}<0.05)$ groups. 